\title{
Stability and Hopf bifurcation analysis of fractional order nonlinear financial system with time delay
}

SANTOSHI PANIGRAHI ${ }^{1}$, Sunita Chand ${ }^{1}$, and S Balamuralitharan ${ }^{2}$

${ }^{1}$ Siksha O Anusandhan University

${ }^{2}$ SRM Institute of Science and Technology

March 6, 2021

\begin{abstract}
In this paper, we study a fractional order time delay for nonlinear financial system. By using Laplace transformation, stability and Hopf bifurcation analysis have been done for the model. Furthermore, numerical simulation has been carried out for better understanding of our results.
\end{abstract}

\section{Hosted file}

Nonlinear Finance Model.pdf available at https://authorea.com/users/399918/articles/512314stability-and-hopf-bifurcation-analysis-of-fractional-order-nonlinear-financial-systemwith-time-delay 
figures/s1/s1-eps-converted-to.pdf 
figures/s2/s2-eps-converted-to.pdf 
figures/s3/s3-eps-converted-to.pdf 
figures/s4/s4-eps-converted-to.pdf 
figures/s5/s5-eps-converted-to.pdf 
figures/s6/s6-eps-converted-to.pdf 
figures/u1/u1-eps-converted-to.pdf 
figures/u2/u2-eps-converted-to.pdf 
figures/u3/u3-eps-converted-to.pdf 
figures/u4/u4-eps-converted-to.pdf 
figures/u5/u5-eps-converted-to.pdf 
figures/u6/u6-eps-converted-to.pdf 